\title{
Observation of dispersive wave emission by temporal cavity solitons
}

\author{
Jae K. Jang, ${ }^{*}$ Miro Erkintalo, Stuart G. Murdoch, and Stéphane Coen \\ Physics Department, The University of Auckland, Private Bag 92019, Auckland 1142, New Zealand \\ ${ }^{*}$ Corresponding author: jake.jang.ur.mate@gmail.com
}

Compiled March 14, 2018

\begin{abstract}
We examine a coherently-driven, dispersion-managed, passive Kerr fiber ring resonator and report the first direct experimental observation of dispersive wave emission by temporal cavity solitons. Our observations are in excellent agreement with analytical predictions and they are fully corroborated by numerical simulations. These results lead to a better understanding of the behavior of temporal cavity solitons under conditions where higher-order dispersion plays a significant role. Significantly, since temporal cavity solitons manifest themselves in monolithic microresonators, our results are likely to explain the origins of spectral features observed in broadband Kerr frequency combs. (c) 2018 Optical Society of America

OCIS codes: (190.5530) Pulse propagation and temporal solitons; (190.4370) Nonlinear optics, fibers; (140.4780) Optical resonators.
\end{abstract}

The emission of dispersive waves (DWs) by intense light pulses perturbed by high-order dispersion has been studied for decades, and it is well documented in nonlinear fiber optics [1-3]. It typically occurs when the phase of a propagating pulse evolves identically to the phase of a linear wave (or waves) at a different frequency, allowing this wave to be amplified through resonant energy transfer. The vast majority of studies associate DW emission exclusively to bright solitons, but the process is in fact much more general: it can occur with dark solitons [4] and even with non-solitonic pulses both in the normal and in the anomalous dispersion regimes [5-7]. Today the process is recognized as one of the central frequency conversion mechanisms in nonlinear fiber optics, where it has been harnessed to realize tunable sources of coherent radiation [8, 9], and identified as a critical component in the generation of broadband supercontinua [10,11].

DWs have now also started to be put forward in another broadband frequency generation paradigm, namely the generation of frequency combs from high-Q Kerr microresonators. This topic has been under intense experimental investigation since 2007 [12, 13], and recent progresses in theoretical modeling have identified some spectral features of these socalled "Kerr combs" as possibly arising from DW emission [5, 14, 15]. More specifically, in some conditions, Kerr combs are now being understood to be underlined in the time domain by dissipative structures known as temporal cavity solitons (CSs) [14, 16, 17]. It is these short CS pulses, and the related periodic modulational instability (MI) patterns, that appear to emit DWs under low dispersion conditions, with temporal instabilities - when present - responsible for additional spectral broadening of the DW peak [18, 19]. This proposition is further supported by the fact that DW emission can be linked to cascaded four-wave mixing [5], the frequency-domain process underlying Kerr comb generation [12,13].

Temporal CSs have just been reported in Kerr comb microresonator experiments [20] but an inconvenient fact remains: temporal CSs have never been observed to emit DWs in any platform. This shortfall can be explained by noting that, so far, all experiments conclusively involving temporal CSs have been carried out far from any zero-dispersion wavelength (ZDW) [16, 20, 21], i.e., in conditions where higherorder dispersion plays a negligible role [10]11]. In this Letter, we address this point by studying experimentally temporal CSs, for the first time in a low-dispersion cavity. Our study is performed in a single-mode fiber loop, in which we can more reliably control the excitation of temporal CSs and the dispersion characteristics of the resonator compared to a microresonator. DWs are positively identified in our experiment and their emission wavelength found to agree with a recent theoretical model [22].

Our experimental set-up is illustrated in Fig. 1 and is similar to that used in previous temporal CS experiments [16,21]. The cavity is coherently driven by a continuous-wave (cw) ultra-narrow linewidth $(<1 \mathrm{kHz}) \mathrm{DFB}$ fiber laser at $1550 \mathrm{~nm}$ wavelength, which is amplified to $1-1.3 \mathrm{~W}$. A band-pass filter (BPF) rejects the noise from the optical amplifier before the driving beam is injected into the cavity through a 90/10 fiber coupler. Our cavity incorporates an optical fiber isolator which prevents the build-up of stimulated Brillouin scattering (SBS), a wavelength division multiplexer (WDM) to couple the addressing pulses used to excite the CSs, a 99/1 tap coupler through which the output spectra are monitored

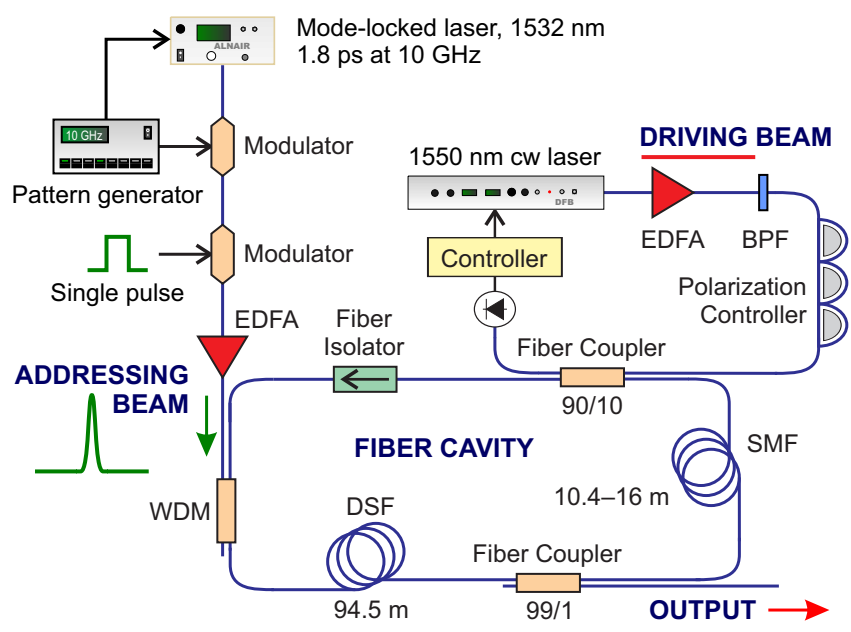

Fig. 1. A schematic of our experimental set-up. EDFA: Erbium-doped fiber amplifier, BPF: band-pass filter, WDM: wavelength division multiplexer, SMF: single-mode fiber, and DSF: dispersion-shifted fiber. 
with an optical spectrum analyzer, and some extra length of fiber. The overall cavity finesse $\mathscr{F}=\pi / \alpha$ is about 18 , with $\alpha=0.176$ representing half the percentage of power loss per round-trip. A PID feedback control loop actuating the laser frequency locks the driving laser near a cavity resonance and keeps the system interferometrically stable. The error signal of the control loop is derived from the part of the driving power that is reflected off the input coupler. In practice we operate the laser with a detuning $\delta_{0}=0.5-0.56$ rad before the closest cavity resonance. To excite temporal CSs, we used the same incoherent writing process as used in previous experiments [16, 21], namely cross-phase modulation between the $\mathrm{cw}$ intracavity field and external addressing pulses. The addressing pulses were $1.8 \mathrm{ps}$ long and generated by a $1532 \mathrm{~nm}-$ wavelength mode-locked laser with a $10 \mathrm{GHz}$ repetition rate. These pulses were selected by a series of two intensity modulators (see [21]) before being amplified and launched in the cavity through the WDM coupler. For each measurement, the cavity was filled with about 1300 temporal CSs. As all the CSs that circulate in our cavity are identical [16], this simply boosts the spectral power of the CS component relative to the $\mathrm{cw}$ background, improving the quality of our measurements.

The fiber path around the cavity is composed of $94.5 \mathrm{~m}$ of dispersion-shifted fiber (DSF) and $10.4 \mathrm{~m}$ to $16.0 \mathrm{~m}$ of standard single-mode fiber (SMF). Adjusting the length of the SMF segment allows us to vary the average cavity dispersion, and to explore CS dynamics in the regime where the third-order dispersive perturbation plays a significant role. The combination of two fibers is justified by the relatively high-finesse: it implies that the field does not vary significantly over the course of a single round-trip (the cavity photon lifetime being much larger than the round-trip time) and therefore that the lumped aspect of the cavity is not so relevant. The round-trip-averaged dispersion and nonlinearity are instead the important quantities [23]. At our operating wavelength, the DSF exhibits normal group-velocity dispersion (GVD), with 2nd- and 3rd-order dispersion coefficients $\beta_{2}^{\mathrm{DSF}}=1.95 \mathrm{ps}^{2} / \mathrm{km}$ and $\beta_{3}^{\mathrm{DSF}}=0.2 \mathrm{ps}^{3} / \mathrm{km}$, while the SMF presents anomalous dispersion with the well-known values $\beta_{2}^{\mathrm{SMF}}=-20 \mathrm{ps}^{2} / \mathrm{km}$ and $\beta_{3}^{\mathrm{SMF}}=0.1 \mathrm{ps}^{3} / \mathrm{km}$. Based on these parameters, varying the length of the SMF segment changes the average GVD of our cavity $\left\langle\beta_{2}\right\rangle$ from -0.23 to $-1.23 \mathrm{ps}^{2} / \mathrm{km}$. At the same time $\left\langle\beta_{3}\right\rangle$ stays relatively constant (to better than $3 \%$ ) at $0.19 \mathrm{ps}^{3} / \mathrm{km}$. Clearly, the relative contribution of third-order dispersion with respect to secondorder dispersion can thus be controlled in our system. To quantify this, we define a normalized third-order dispersion coefficient $d_{3}=\sqrt{2 \alpha / L} \beta_{3} /\left(3\left|\beta_{2}\right|^{3 / 2}\right)$ where $L$ is the total length of the cavity. The normalization is such that the cavity field decay rate and the GVD coefficient are both set to -1 [24,25]. When $d_{3} \ll 1$ (respectively, $\gg 1$ ), 2nd (3rd) order dispersion dominates, and when $d_{3} \sim 1$ the two contribute comparably. Finally, we estimate the average nonlinear coefficient of the cavity to be about $\gamma=1.9 \mathrm{~W}^{-1} \mathrm{~km}^{-1}$.

In addition to experiments we also numerically simulate the nonlinear cavity dynamics. Our simulations use a full lumped model, in which each cavity element is separately taken into account. Propagation through each fiber segment
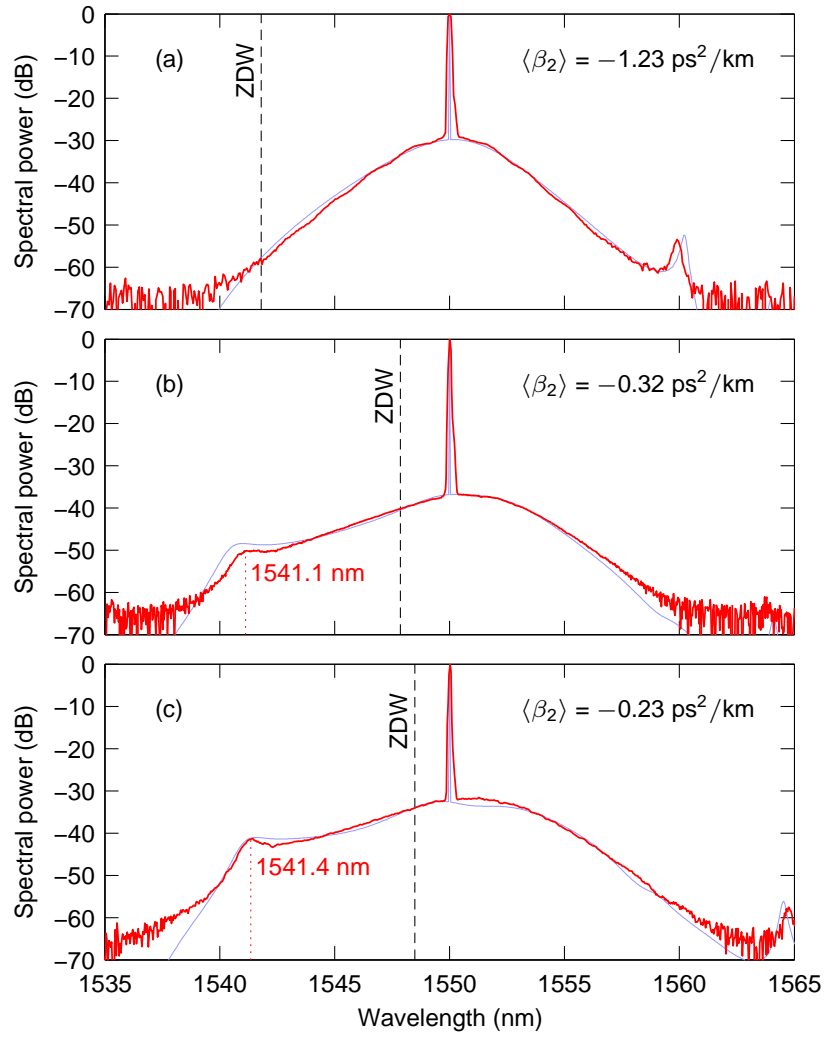

Fig. 2. Experimental (red) and numerical (blue) spectra of temporal CSs for three different values of average cavity dispersion as shown in each figure. In each case, the black dashed line indicates the position of the ZDW while the red dotted line highlights the observed DW wavelength. (a) $P_{\text {in }}=1 \mathrm{~W}, \delta_{0}=0.55 \mathrm{rad} ;(\mathrm{b}) P_{\text {in }}=1 \mathrm{~W}, \delta_{0}=0.5 \mathrm{rad}$; (c) $P_{\text {in }}=1.3 \mathrm{~W}, \delta_{0}=0.56 \mathrm{rad}$.

is modeled using a generalized nonlinear Schrödinger equation [10], the driving field is interferometrically added at the beginning of each roundtrip, and the remaining discrete losses and/or components are modeled through the use of localized transmission functions.

Figures 2a)-(c) show experimental (red) and numerical (blue) spectra of temporal CSs obtained for three different averaged cavity dispersion. We first note the overall remarkable agreement between the simulations and the experiments. Results in Fig.2 (2) correspond to a cavity with 16 m of SMF, or equivalently an average 2nd-order dispersion coefficient $\left\langle\beta_{2}\right\rangle=-1.23 \mathrm{ps}^{2} / \mathrm{km}$. The spectrum is typical of a temporal CS: the broad spectral wings are associated with the narrow temporal pulsed structure of the soliton, while the central narrow "DC" peak highlights the presence of the cw background on which temporal CSs are superimposed [16]. Although the dispersion is already relatively low, the CS spectrum does not extend significantly past the ZDW (highlighted as a dashed line in Fig. (2), and no significant spectral features are present in the normal dispersion regime where DWs would be expected. This is attributed to the fact that the contribution from 3 rd-order dispersion is still small compared to $\beta_{2}$, and indeed the normalized 3rd-order dispersion coefficient is very small, $d_{3} \simeq 0.08 \ll 1$. The only noticeable feature seen in Fig. 2 (a) 
is a quasi-phase-matched Kelly sideband at $1560 \mathrm{~nm}$ in the anomalous dispersion regime. It arises due to periodic nature of our cavity which is made up of two different fiber types [26]. Consequently, we note that the mean-field model that is usually employed to describe CSs [14, 16, 27] does not account for this Kelly sideband. A full lumped cavity model as we have been using is required instead.

As the average dispersion is reduced to $-0.32 \mathrm{ps}^{2} / \mathrm{km}$ (corresponding to $10.9 \mathrm{~m}$ of SMF, and to $d_{3} \simeq 0.64$ ), the spectrum develops a peak at $1541.1 \mathrm{~nm}$ in the normal dispersion regime [Fig. 2[b)]. The peak shifts slightly to $1541.4 \mathrm{~nm}$ and becomes more prominent [Fig. 2 (c)] at an even lower average dispersion of $-0.23 \mathrm{ps}^{2} / \mathrm{km}$ (corresponding to $10.4 \mathrm{~m}$ of $\mathrm{SMF}$, and to $d_{3} \simeq 1.05$ ). This peak is a clear signature of DWs emitted by the circulating temporal CSs, and the trend observed when the average dispersion reduces fully agrees with this conclusion. Indeed, as we reduce the average GVD coefficient $\left\langle\beta_{2}\right\rangle$, we are effectively shifting the ZDW towards the driving beam wavelength. This pushes the DW towards the driving beam, leading to a more efficient amplification [3].

To more quantitatively establish that the spectral feature seen in Figs. 2(b)-(c) corresponds to a DW, we must show that its wavelength obeys the relevant phase-matching condition. In some cases, especially when broadband Kerr comb generation takes place in high-Q microresonators, the known condition for conservative solitons [3] has been shown to provide a good approximation [14]. However, this limit does not apply here and a more general prediction requires the cavity geometry, and in particular the effect of the cavity detuning, to be fully taken into account. The theoretical study of Ref. [22] has recently addressed this issue based on the following argument: In the cavity configuration, because of the dissipative losses, the DW is a stationary localized radiation tail attached to the CS. The DW frequency can thus be found by examining how this tail asymptotically approaches the cw background on which the temporal CSs are superimposed. Specifically, one considers the ansatz $E=E_{0}+a \exp (-i Q \tau)+b \exp \left(i Q^{*} \tau\right)$ (the $*$ denoting complex conjugation) in the mean-field model of the cavity [14, 16, 27, 28], with $\tau$ the fast-time describing the temporal profile of the field inside the cavity and $E_{0}$ the cw lower state background, and one looks for a self-consistent, stationary, linearized $\left(|a|,|b| \ll\left|E_{0}\right|\right)$ DW solution. This leads to the following condition for the complex frequency $Q$ :

$$
\begin{aligned}
-\alpha+ & i \frac{\beta_{3} L}{3 !} Q^{3}-i V Q \\
& \pm i \sqrt{\left(2 \gamma L P_{0}-\delta_{0}+\frac{\beta_{2} L}{2 !} Q^{2}\right)^{2}-\left(\gamma L P_{0}\right)^{2}}=0 .
\end{aligned}
$$

Here $P_{0}=\left|E_{0}\right|^{2}$ is the power level of the cw lower state background, while $V$ is a drift velocity that accounts for the fact that 3rd-order dispersion (or any odd order of dispersion) makes the CSs group-velocity slightly different from that of the driving field [23, 24]. This is associated with a spectral shift of the CS spectrum with respect to the driving frequency and in [22] has been interpreted as resulting from the spectral recoil due to the emission of DWs. Specifically, $V$ repre- sents the group-delay accumulated by the temporal CSs with respect to the driving field over one round-trip (and has the units of time). We note that the above equation can be generalized to include arbitrary orders of dispersion by adding the extra even (odd) order dispersion terms in the form $\beta_{k} L Q^{k} / k$ ! (for $k \geq 4$ ) to the $\beta_{2}\left(\beta_{3}\right)$ contributions, respectively.

In considering Eq. (1), we can restrict ourselves to the + sign alternative in front of the square root because the solutions of the two cases are related by the transformation $Q \rightarrow-Q^{*}$. This fact highlights that, in the cavity geometry, DW peaks should appear in pairs symmetrically located with respect to the driving frequency. Further analysis of the eigenvectors of the problem [22] indicates however that in our experiment the dominant DW is always up-frequency shifted. The down-frequency shifted symmetrical counterpart is predicted to be significantly weaker, and so far we have been unable to detect it.

Equation (11) was studied in [22] with the loss coefficient $\alpha$ neglected, which was appropriate for the context of highQ microresonators pertinent to that work. In that condition, the solutions $Q$ are purely real (and directly interpreted as the angular frequency shift of the DW with respect to the driving frequency). In our fiber cavity experiment, the losses are more important but we can still simplify Eq. (1) by noting that with our measured background power level $P_{0} \simeq 500-600 \mathrm{~mW}$, the nonlinear round-trip phase shift $\gamma L P_{0} \sim 0.1 \mathrm{rad}$, making the second-term in the square root, $\left(\gamma L P_{0}\right)^{2}$, negligible. Eq. (1) then becomes a polynomial in $Q$,

$$
\frac{\beta_{3} L}{3 !} Q^{3}+\frac{\beta_{2} L}{2 !} Q^{2}-V Q+\left[\left(2 \gamma L P_{0}-\delta_{0}\right)+i \alpha\right]=0 .
$$

In the form above, Eq. (2) can be easily compared to the classical DW phase-matching condition of conservative solitons [2,3] and the differences with the cavity geometry readily identified. We note in particular that the peak power of the temporal CS does not play any direct role: rather, it is the power of the CS background $P_{0}$ which is relevant. When the dispersion terms $\beta_{k}$ dominate, Eq. (2) approaches the conservative soliton expression [14].

In order to solve Eq. (2) for the parameters of our experiment, the only thing we lack is the CS drift velocity $V$. We can obtain this quantity from the observed spectral recoil of the CS spectrum. In Fig. 2 (c), the peak of the CS spectral components does not coincide with the driving wavelength but rather is shifted towards longer wavelengths by about $1 \mathrm{~nm}$ [corresponding to a frequency shift $\Delta \Omega_{\mathrm{CS}} /(2 \pi)=$ $-125 \mathrm{GHz}$. This shift translates into an extra group delay of $V=\left\langle\beta_{2}\right\rangle \Delta \Omega_{\mathrm{CS}} L+\left\langle\beta_{3}\right\rangle \Delta \Omega_{\mathrm{CS}}^{2} L / 2 \simeq 25$ fs per round-trip for the CS. The situation is not as clear for the spectrum of Fig. 2(b), but we estimate $V$ to be less than half that value in this case. With these values, the dispersion and loss coefficients quoted above, and the detuning and driving power levels stated in the caption of Fig. 2] (which then determine the intracavity $\mathrm{cw}$ background power level $P_{0}$ based on the cavity mean-field model), Eq. (2) predicts the emission of DWs at wavelengths (derived from the real part of $Q$ ) of 1540.9$1541.2 \mathrm{~nm}$ (considering $V$ within $0-12.5 \mathrm{fs}$ ) for Fig. 2](b), and $1541.3 \mathrm{~nm}$ for Fig. 2 (c). These predictions are in very good 


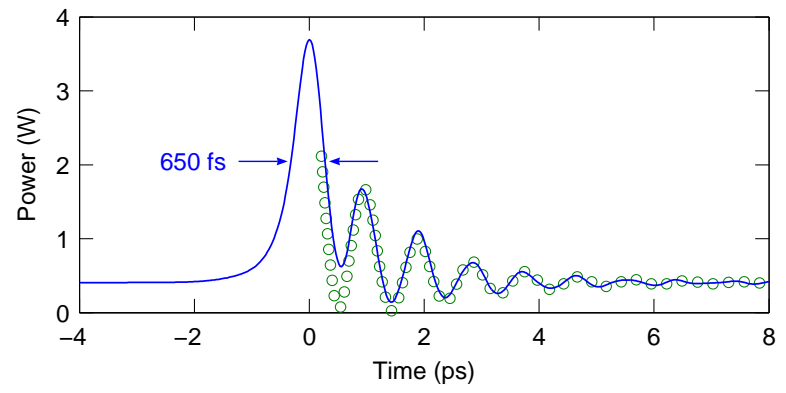

Fig. 3. Simulated intensity profile (blue) of the temporal CS corresponding to the spectrum shown in light-blue in Fig. 22 (c), superimposed with the DW tail calculated with the analytical asymptotic analysis (green circles).

agreement with experiments and further support our interpretation of the observed spectral peaks as genuine DWs.

Because the cavity losses are included in our analysis, the solutions of Eq. (2) are complex. By examining the ansatz, it must be clear that the imaginary part of $Q$ is related to the time-constant with which the dispersive radiation tail of the temporal CS exponentially decays to the background. We have unfortunately been unable to temporally resolve this radiation in our experiment. Still, in order to provide some form of comparison, we have plotted in Fig. 3 the intensity profile of the temporal CS (blue) corresponding to the simulated (light-blue curve) spectrum plotted in Fig.2 (c), superimposed with the analytically predicted DW tail (green circles). The latter corresponds to the expression $\left|E_{0}+a \exp (-i Q \tau)\right|^{2}$ (ignoring the weak down-frequency shifted component $b$ of the ansatz), with $Q=2 \pi(1.07 \mathrm{THz})-i /(1.54 \mathrm{ps})$ the solution of Eq. (2), and we used the complex DW amplitude $a$ as a fit parameter. Clearly the agreement is excellent. Compounded by the good agreement between the simulated and experimental data in Fig. 2, this strongly supports that we have observed a temporal CS with a main peak of about 650 fs duration, and with an oscillating DW tail decaying (in intensity) over a timescale of about 3 ps.

In conclusion, we have directly observed experimentally the emission of DWs by temporal CSs. Our experiment was performed in a dispersion-managed single-mode fiber ring resonator and the DWs manifest themselves as spectral peaks that develop across the ZDW when the average cavity dispersion is sufficiently reduced. The peak becomes more prominent and shifts closer to the driving beam wavelength with decreasing dispersion, in agreement with theoretical analysis [22]. Our observations further reinforce the case that Kerr combs generated in microresonators are in some conditions underlined by temporal CSs, and could explain the very similar spectral peaks observed in this context [14, 18, 19].

This work was supported by the Marsden Fund Council from Government funding, administered by the Royal Society of New Zealand.

\section{References}

1. P. K. A. Wai, C. R. Menyuk, Y. C. Lee, and H. H. Chen, Opt. Lett. 11, 464 (1986).
2. P. K. A. Wai, H. H. Chen, and Y. C. Lee, Phys. Rev. A 41, 426 (1990).

3. N. Akhmediev and M. Karlsson, Phys. Rev. A 51, 2602 (1995).

4. V. I. Karpman, Phys. Lett. A 181, 211 (1993).

5. M. Erkintalo, Y. Q. Xu, S. G. Murdoch, J. M. Dudley, and G. Genty, Phys. Rev. Lett. 109, 223904 (2012).

6. K. E. Webb, Y. Q. Xu, M. Erkintalo, and S. G. Murdoch, Opt. Lett. 38, 151 (2013).

7. M. Conforti and S. Trillo, Opt. Lett. 38, 3815 (2013).

8. G. Chang, L.-J. Chen, and F. X. Kärtner, Opt. Lett. 35, 2361 (2010).

9. K. F. Mak, J. C. Travers, P. Hölzer, N. Y. Joly, and P. St. J. Russell, Opt. Express 21, 10942 (2013).

10. J. M. Dudley, G. Genty, and S. Coen, Rev. Mod. Phys. 78, 1135 (2006).

11. D. V. Skryabin and A. V. Gorbach, Rev. Mod. Phys. 82, 1287 (2010).

12. P. Del'Haye, A. Schliesser, O. Arcizet, T. Wilken, R. Holzwarth, and T. J. Kippenberg, Nature 450, 1214 (2007).

13. T. J. Kippenberg, R. Holzwarth, and S. A. Diddams, Science 332, 555 (2011).

14. S. Coen, H. G. Randle, T. Sylvestre, and M. Erkintalo, Opt. Lett. 38, 37 (2013).

15. M. R. E. Lamont, Y. Okawachi, and A. L. Gaeta, Opt. Lett. 38, 3478 (2013).

16. F. Leo, S. Coen, P. Kockaert, S.-P. Gorza, Ph. Emplit, and M. Haelterman, Nat. Photon. 4, 471-476 (2010).

17. S. Coen and M. Erkintalo, Opt. Lett. 38, 1790 (2013).

18. Y. Okawachi, K. Saha, J. S. Levy, Y. H. Wen, M. Lipson, and A. L. Gaeta, Opt. Lett. 36, 3398 (2011).

19. M. Erkintalo and S. Coen, Opt. Lett. 39, 283 (2014).

20. T. Herr, V. Brasch, J. D. Jost, C. Y. Wang, N. M. Kondratiev, M. L. Gorodetsky, and T. J. Kippenberg, Nature Photon. 8, 145 (2014).

21. J. K. Jang, M. Erkintalo, S. G. Murdoch, and S. Coen, Nature Photon. 7, 657 (2013).

22. C. Milián and D. V. Skryabin, Opt. Express 22, 3732 (2014).

23. F. Leo, A. Mussot, P. Kockaert, Ph. Emplit, M. Haelterman, and M. Taki, Phys. Rev. Lett. 110, 104103 (2013).

24. P. Parra-Rivas, D. Gomila, F. Leo, S. Coen, and L. Gelens, Opt. Lett. 39, 2971 (2014).

25. Y. Xu and S. Coen, Opt. Lett. 39, 3492 (2014).

26. S. M. J. Kelly, Electron. Lett. 28, 806 (1992).

27. L. A. Lugiato and R. Lefever, Phys. Rev. Lett. 58, 2209 (1987).

28. Y. K. Chembo and C. R. Menyuk, Phys. Rev. A 87, 053852 (2013). 


\section{References with titles}

\section{References}

1. P. K. A. Wai, C. R. Menyuk, Y. C. Lee, and H. H. Chen, "Nonlinear pulse propagation in the neighborhood of the zerodispersion wavelength of monomode optical fibers," Opt. Lett. 11, 464-466 (1986).

2. P. K. A. Wai, H. H. Chen, and Y. C. Lee, "Radiations by 'solitons' at the zero group-dispersion wavelength of single-mode optical fibers," Phys. Rev. A 41, 426-439 (1990).

3. N. Akhmediev and M. Karlsson, "Cherenkov radiation emitted by solitons in optical fibers," Phys. Rev. A 51, 2602-2607 (1995).

4. V. I. Karpman, "Stationary and radiating dark solitons of the third order nonlinear Schrödinger equation," Phys. Lett. A 181, 211-215 (1993).

5. M. Erkintalo, Y. Q. Xu, S. G. Murdoch, J. M. Dudley, and G. Genty, "Cascaded phase matching and nonlinear symmetry breaking in fiber frequency combs," Phys. Rev. Lett. 109, 223904/1-5 (2012).

6. K. E. Webb, Y. Q. Xu, M. Erkintalo, and S. G. Murdoch, "Generalized dispersive wave emission in nonlinear fiber optics," Opt. Lett. 38, 151-153 (2013).

7. M. Conforti and S. Trillo, "Dispersive wave emission from wave breaking," Opt. Lett. 38, 3815-3818 (2013).

8. G. Chang, L.-J. Chen, and F. X. Kärtner, "Highly efficient Cherenkov radiation in photonic crystal fibers for broadband visible wavelength generation," Opt. Lett. 35, 2361-2363 (2010).

9. K. F. Mak, J. C. Travers, P. Hölzer, N. Y. Joly, and P. St. J. Russell, "Tunable vacuum-UV to visible ultrafast pulse source based on gas-filled Kagome-PCF," Opt. Express 21, 10942 10953 (2013).

10. J. M. Dudley, G. Genty, and S. Coen, "Supercontinuum generation in photonic crystal fiber," Rev. Mod. Phys. 78, 1135-1184 (2006).

11. D. V. Skryabin and A. V. Gorbach, "Colloquium: Looking at a soliton through the prism of optical supercontinuum," Rev. Mod. Phys. 82, 1287-1299 (2010).

12. P. Del'Haye, A. Schliesser, O. Arcizet, T. Wilken, R. Holzwarth, and T. J. Kippenberg, "Optical frequency comb generation from a monolithic microresonator," Nature 450, 1214-1217 (2007).

13. T. J. Kippenberg, R. Holzwarth, and S. A. Diddams, "Microresonator-based optical frequency combs," Science 332, 555-559 (2011).

14. S. Coen, H. G. Randle, T. Sylvestre, and M. Erkintalo, "Modeling of octave-spanning Kerr frequency combs using a generalized mean-field Lugiato-Lefever model," Opt. Lett. 38, 37-39 (2013).

15. M. R. E. Lamont, Y. Okawachi, and A. L. Gaeta, "Route to stabilized ultrabroadband microresonator-based frequency combs," Opt. Lett. 38, 3478-3481 (2013).

16. F. Leo, S. Coen, P. Kockaert, S.-P. Gorza, Ph. Emplit, and M. Haelterman, "Temporal cavity solitons in one-dimensional Kerr media as bits in an all-optical buffer," Nature Photon. 4, 471-476 (2010).

17. S. Coen and M. Erkintalo, "Universal scaling laws of Kerr frequency combs," Opt. Lett. 38, 1790-1792 (2013).

18. Y. Okawachi, K. Saha, J. S. Levy, Y. H. Wen, M. Lipson, and A. L. Gaeta, "Octave-spanning frequency comb generation in a silicon nitride chip," Opt. Lett. 36, 3398-3400 (2011).
19. M. Erkintalo and S. Coen, "Coherence properties of Kerr frequency combs," Opt. Lett. 39, 283-286 (2014).

20. T. Herr, V. Brasch, J. D. Jost, C. Y. Wang, N. M. Kondratiev, M. L. Gorodetsky, and T. J. Kippenberg, "Temporal solitons in optical microresonators," Nat. Photon. 8, 145-152 (2014).

21. J. K. Jang, M. Erkintalo, S. G. Murdoch, and S. Coen, "Ultraweak long-range interactions of solitons observed over astronomical distances," Nature Photon. 7, 657-663 (2013).

22. C. Milián and D. V. Skryabin, "Soliton families and resonant radiation in a micro-ring resonator near zero group-velocity dispersion," Opt. Express 22, 3732-3739 (2014).

23. F. Leo, A. Mussot, P. Kockaert, Ph. Emplit, M. Haelterman, and M. Taki, "Nonlinear symmetry breaking induced by thirdorder dispersion in optical fiber cavities," Phys. Rev. Lett. 110, 104103 (2013).

24. P. Parra-Rivas, D. Gomila, F. Leo, S. Coen, and L. Gelens, "Third-order chromatic dispersion stabilizes Kerr frequency combs," Opt. Lett. 39, 2971-2974 (2014).

25. Y. Xu and S. Coen, "Experimental observation of the spontaneous breaking of the time-reversal symmetry in a synchronously pumped passive Kerr resonator," Opt. Lett. 39, 3492-3495 (2014).

26. S. M. J. Kelly, "Characteristic sideband instability of periodically amplified average soliton," Electron. Lett. 28, 806-807 (1992).

27. L. A. Lugiato and R. Lefever, "Spatial dissipative structures in passive optical systems," Phys. Rev. Lett. 58, 2209-2211 (1987).

28. Y. K. Chembo and C. R. Menyuk, "Spatiotemporal LugiatoLefever formalism for Kerr-comb generation in whisperinggallery-mode resonators," Phys. Rev. A 87, 053852 (2013). 